\title{
Low-energy excitations and quasielastic contribution to electron-nucleus and neutrino-nucleus scattering in the continuum random-phase approximation
}

\author{
V. Pandey, ${ }^{*}$ N. Jachowicz, ${ }^{\dagger}$ T. Van Cuyck, J. Ryckebusch, and M. Martini \\ Department of Physics and Astronomy, Ghent University, Proeftuinstraat 86, B-9000 Ghent, Belgium \\ (Received 15 December 2014; revised manuscript received 16 July 2015; published 7 August 2015)
}

\begin{abstract}
We present a detailed study of a continuum random-phase approximation approach to quasielastic electronnucleus and neutrino-nucleus scattering. We compare the $\left(e, e^{\prime}\right)$ cross-section predictions with electron scattering data for the nuclear targets ${ }^{12} \mathrm{C},{ }^{16} \mathrm{O}$, and ${ }^{40} \mathrm{Ca}$, in the kinematic region where quasielastic scattering is expected to dominate. We examine the longitudinal and transverse contributions to ${ }^{12} \mathrm{C}\left(e, e^{\prime}\right)$ and compare them with the available data. We find an overall satisfactory description of the $\left(e, e^{\prime}\right)$ data. Further, we study the ${ }^{12} \mathrm{C}\left(v_{\mu}, \mu^{-}\right)$cross sections relevant for accelerator-based neutrino-oscillation experiments. We pay special attention to low-energy excitations which can account for non-negligible contributions in measurements, and require a beyond-Fermi-gas formalism.
\end{abstract}

DOI: 10.1103/PhysRevC.92.024606

PACS number(s): 25.30.Pt, 13.15.+g, 24.10.Cn, 21.60.Jz

\section{INTRODUCTION}

The quest for a completion of our knowledge of neutrinooscillation parameters has made tremendous progress in recent years. Still, neutrino-oscillation experiments face a number of challenges. Major issues are the identification of the basic processes contributing to the neutrino-nucleus signal in a detector and the reduction of the systematic uncertainties. A thorough understanding of the complexity of the nuclear environment and its electroweak response at low and intermediate energies is required. Charged-current quasielastic (CCQE) processes account for a large share of the detected signals in many experiments. Although several cross-section measurements have been performed [1-7], uncertainties connected to the electroweak responses persist [8,9].

Despite substantial progress in the understanding of the different processes involved in the signal of neutrino-oscillation experiments, the simulation codes are primarily based on a Fermi-gas description of the nucleus. Relativistic Fermi-gas (RFG) based models are employed in Monte Carlo event generators. The RFG model provides a basic picture of the nucleus as a system of quasifree nucleons and takes into account the Fermi motion and Pauli blocking effects. The analysis of electron-scattering data suggests that at momentum transfers $q \approx 500 \mathrm{MeV} / c$, the RFG model describes the general behavior of the quasielastic $(\mathrm{QE})$ cross section sufficiently accurately, but its description becomes poor for smaller momentum transfers, where nuclear effects are more prominent. Since the neutrino flux in the oscillation experiments is distributed over energies from very low to a few $\mathrm{GeV}$, the cross section picks up contributions from all energies. The low-excitation-energy cross sections do not receive proper attention in an RFG description. Furthermore, even at higher incoming neutrino energy, the contributions stemming from low transferred energies are not negligible. At low energy transfers, the nuclear structure certainly needs

\footnotetext{
*Vishvas.Pandey@UGent.be

†Natalie.Jachowicz@UGent.be
}

a beyond RFG description. Several studies emphasizing the low energy excitation in the framework of neutrino-nuclear interactions [10-14] have been performed. Those studies, however, have not been explicitly extended to explore the kinematics of MiniBooNE [15], T2K [16], and other similar experiments.

In this paper, we present a continuum random-phase approximation (CRPA) approach for the description of QE electroweak scattering off the nucleus, crucial for acceleratorbased neutrino-oscillation experiments. We pay special attention to low-energy nuclear excitations. In this context, the availability of a large amount of high-precision electronnucleus scattering data is of the utmost importance, as it allows one to test the reliability of the reaction model.

Several models have been developed to study electronnucleus scattering and further generalized to describe neutrinonucleus cross sections [17-34]. An extensive test against the inclusive quasielastic electron scattering is performed within an RFG and plane-wave impulse approximation approach in Ref. [30], while a spectral function based approach is assessed in Ref. [26]. The model we adopt takes a Hartree-Fock (HF) description of nuclear dynamics as a starting point and additionally implements long-range correlations through a CRPA framework with an effective Skyrme nucleon-nucleon twobody interaction. We solve the CRPA equations by a Green's function approach. Thereby, the polarization propagator is approximated by an iteration of its first-order contribution. In this way, the formalism implements the description of one-particle one-hole excitations out of the correlated nuclear ground state. To improve our description of the kinematics of the interaction at intermediate energies, we implemented an effective relativistic approach proposed in Refs. [27,28,35].

The article is organized as follows. In Sec. II, we outline the details of the QE electron and neutrino-nucleus cross-section formalism. We describe the CRPA framework for calculating nuclear responses. Section III is divided into two parts: In Sec. III A, we present numerical results of electron-scattering cross sections (on a variety of nuclear targets) and responses (on ${ }^{12} \mathrm{C}$ ) and compare them with the available data. In Sec. III B, we discuss neutrino-scattering results in the context 
of accelerator-based neutrino-oscillation experiments. We pay special attention to low-energy neutrino-induced nuclear excitations. Conclusions can be found in Sec. IV.

\section{FORMALISM}

In this section, we describe our CRPA-based approach for the calculation of the nuclear response for inclusive electron and neutrino-nucleus scattering in the QE region. This approach was successful in describing exclusive photo-induced and electron-induced $\mathrm{QE}$ processes [36,37], and inclusive neutrino scattering at supernova energies [38-43]. We have also used this approach to calculate the inclusive CCQE antineutrino-nucleus scattering cross sections at intermediate energies [44]. Here, we are using an updated version of the same formalism.

We consider QE electron and CCQE neutrino scattering off a nucleus under conditions where the details of the final hadron state remain unobserved. As shown in Fig. 1, an incident electron (neutrino) with four-momentum $E_{i}, \vec{k}_{i}\left(\varepsilon_{i}, \vec{\kappa}_{i}\right)$ scatters off a nucleus via the exchange of a photon ( $W$ boson) and only the outgoing charged lepton with four-momentum $E_{f}, \vec{k}_{f}$ $\left(\varepsilon_{f}, \vec{\kappa}_{f}\right)$ is detected in the final state

$$
e\left(E_{i}, \vec{k}_{i}\right)+A \rightarrow e^{\prime}\left(E_{f}, \vec{k}_{f}\right)+X,
$$

and

$$
v_{l}\left(\varepsilon_{i}, \vec{\kappa}_{i}\right)+A \rightarrow l^{-}\left(\varepsilon_{f}, \vec{\kappa}_{f}\right)+X,
$$

where $l$ represents $e, \mu$, or $\tau$. Further, $A$ is the nucleus in its ground state $\left|J_{i}, M_{i}\right\rangle$ and $X$ is the unobserved hadronic final state.

The double differential cross section for electron and neutrino-nucleus scattering of Eqs. (1) and (2) can be expressed as

$$
\begin{aligned}
\left(\frac{d^{2} \sigma}{d \omega d \Omega}\right)_{e}= & \frac{\alpha^{2}}{Q^{4}}\left(\frac{2}{2 J_{i}+1}\right) E_{f} k_{f} \cos ^{2}(\theta / 2) \\
& \times \zeta^{2}\left(Z^{\prime}, E_{f}, q\right)\left[\sum_{J=0}^{\infty} \sigma_{L, e}^{J}+\sum_{J=1}^{\infty} \sigma_{T, e}^{J}\right],
\end{aligned}
$$

and

$$
\begin{aligned}
\left(\frac{d^{2} \sigma}{d \omega d \Omega}\right)_{\nu}= & \frac{G_{F}^{2} \cos ^{2} \theta_{c}}{(4 \pi)^{2}}\left(\frac{2}{2 J_{i}+1}\right) \varepsilon_{f} \kappa_{f} \\
& \times \zeta^{2}\left(Z^{\prime}, \varepsilon_{f}, q\right)\left[\sum_{J=0}^{\infty} \sigma_{C L, v}^{J}+\sum_{J=1}^{\infty} \sigma_{T, v}^{J}\right],
\end{aligned}
$$

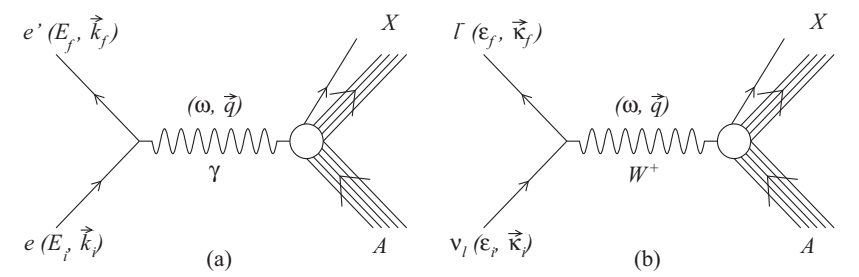

FIG. 1. Inclusive processes considered in this paper: (a) QE electron-nucleus and (b) CCQE neutrino-nucleus $(l=e, \mu, \tau)$, where $X$ is the undetected hadronic final state.

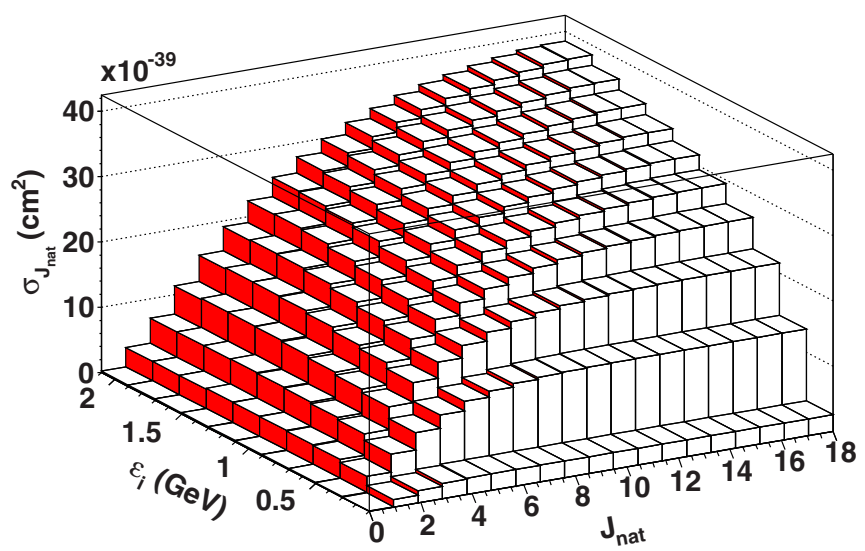

FIG. 2. (Color online) Multipole contributions (for natural parity transitions) to the cross section, as a function of the incoming neutrino energy. The $\sigma_{J_{\text {nat }}}$ denotes the ${ }^{12} \mathrm{C}\left(v_{\mu}, \mu^{-}\right)$cross section including all multipoles of the natural parity excitations up to $J_{\text {nat }}$.

where $\alpha$ is the fine-structure constant, $G_{F}$ is the Fermi coupling constant, and $\theta_{c}$ is the Cabibbo angle. The direction of the outgoing lepton is described by the solid angle $\Omega$. The lepton-scattering angle is $\theta$, the transferred four-momentum is $q^{\mu}(\omega, \vec{q})$, and $Q^{2}=-q_{\mu} q^{\mu}$. Further, $\zeta\left(Z^{\prime}, E, q\right)$ is introduced in order to take into account the distortion of the lepton wave function in the Coulomb field generated by $Z^{\prime}$ protons, within a modified effective momentum approximation [45].

The $\sigma_{L, e}^{J}\left(J\right.$ denotes the multipole number) and $\sigma_{T, e}^{J}$ are the longitudinal and transverse components of the electronnucleus scattering cross section, while $\sigma_{C L, v}^{J}$ and $\sigma_{T, \nu}^{J}$ are the Coulomb-longitudinal and transverse contributions of the neutrino-nucleus scattering cross section. In Fig. 2, we plot the strength obtained by adding the different multipole contributions to the cross section for incident neutrino energies from 0.1 to $2.0 \mathrm{GeV}$. Naturally, the higher the energy of the incident particle, the more multipoles contribute to the cross section. From the figure, one observes that for energies as low as $200 \mathrm{MeV}$, multipoles up to $J=4$ contribute. For energies as high as $2 \mathrm{GeV}$, multipoles up to $J=16$ need to be considered, and the relative weight of small $J$ contributions diminishes.

The (Coulomb) longitudinal and transverse parts of the cross section are both composed of a kinematical factor $v$ and a response function $R$. The response function contains the full nuclear structure information. In the electron-scattering case, the longitudinal $\sigma_{L, e}$ and transverse $\sigma_{T, e}$ components of the cross section can be expressed as follows:

$$
\sigma_{L, e}=v_{e}^{L} R_{e}^{L}, \quad \sigma_{T, e}=v_{e}^{T} R_{e}^{T},
$$

where the leptonic factors $v_{e}^{L}$ and $v_{e}^{T}$ are given by

$$
v_{e}^{L}=\frac{Q^{4}}{|\vec{q}|^{4}}, \quad v_{e}^{T}=\left[\frac{Q^{2}}{2|\vec{q}|^{2}}+\tan ^{2}(\theta / 2)\right] .
$$

Longitudinal $R_{e}^{L}$ and transverse $R_{e}^{T}$ response functions are defined as

$$
\begin{aligned}
& R_{e}^{L}=\left|\left\langle J_{f}|| \widehat{\mathcal{M}}_{J}^{e}(|\vec{q}|)|| J_{i}\right\rangle\right|^{2}, \\
& R_{e}^{T}=\left[\left|\left\langle J_{f}|| \widehat{\mathcal{J}}_{J}^{\mathrm{mag}, e}(|\vec{q}|)|| J_{i}\right\rangle\right|^{2}+\left|\left\langle J_{f}|| \widehat{\mathcal{J}}_{J}^{\mathrm{el}, e}(|\vec{q}|)|| J_{i}\right\rangle\right|^{2}\right] .
\end{aligned}
$$


Here $\widehat{\mathcal{M}}_{J}^{e}, \widehat{\mathcal{J}}_{J}^{\text {mag,e }}$ and $\widehat{\mathcal{J}}_{J}^{\text {el, e }}$ are the longitudinal, transverse magnetic, and transverse electric operators, respectively $[46,47]$. The $\left|J_{i}\right\rangle$ and $\left|J_{f}\right\rangle$ denote the initial and final states of the nucleus.

Similarly for neutrino-scattering processes, we express the Coulomb-longitudinal $\sigma_{C L, v}$ and transverse $\sigma_{T, v}$ parts of the cross section as follows:

$$
\begin{aligned}
\sigma_{C L, v} & =\left[v_{v}^{\mathcal{M}} R_{v}^{\mathcal{M}}+v_{v}^{\mathcal{L}} R_{v}^{\mathcal{L}}+2 v_{v}^{\mathcal{M L}} R_{v}^{\mathcal{M L}}\right], \\
\sigma_{T, v} & =\left[v_{v}^{T} R_{v}^{T}+2 v_{v}^{T T} R_{v}^{T T}\right],
\end{aligned}
$$

where leptonic coefficients $v_{v}^{\mathcal{M}}, v_{v}^{\mathcal{L}}, v_{v}^{\mathcal{M L}}, v_{v}^{T}$, and $v_{v}^{T T}$ are given as

$$
\begin{aligned}
v_{v}^{\mathcal{M}} & =\left[1+\frac{\kappa_{f}}{\varepsilon_{f}} \cos \theta\right], \\
v_{v}^{\mathcal{L}} & =\left[1+\frac{\kappa_{f}}{\varepsilon_{f}} \cos \theta-\frac{2 \varepsilon_{i} \varepsilon_{f}}{|\vec{q}|^{2}}\left(\frac{\kappa_{f}}{\varepsilon_{f}}\right)^{2} \sin ^{2} \theta\right], \\
v_{v}^{\mathcal{M} \mathcal{L}} & =\left[\frac{\omega}{|\vec{q}|}\left(1+\frac{\kappa_{f}}{\varepsilon_{f}} \cos \theta\right)+\frac{m_{l}^{2}}{\varepsilon_{f}|\vec{q}|}\right], \\
v_{v}^{T} & =\left[1-\frac{\kappa_{f}}{\varepsilon_{f}} \cos \theta+\frac{\varepsilon_{i} \varepsilon_{f}}{|\vec{q}|^{2}}\left(\frac{\kappa_{f}}{\varepsilon_{f}}\right)^{2} \sin ^{2} \theta\right], \\
v_{v}^{T T} & =\left[\frac{\varepsilon_{i}+\varepsilon_{f}}{|\vec{q}|}\left(1-\frac{\kappa_{f}}{\varepsilon_{f}} \cos \theta\right)-\frac{m_{l}^{2}}{\varepsilon_{f}|\vec{q}|}\right],
\end{aligned}
$$

and response functions $R_{v}^{\mathcal{M}}, R_{v}^{\mathcal{L}}, R_{v}^{\mathcal{M L}}, R_{v}^{T}$, and $R_{v}^{T T}$ are defined as

$$
\begin{aligned}
R_{v}^{\mathcal{M}} & =\left|\left\langle J_{f}|| \widehat{\mathcal{M}}_{J}^{v}(|\vec{q}|)|| J_{i}\right\rangle\right|^{2} \\
R_{v}^{\mathcal{L}} & =\left|\left\langle J_{f}|| \widehat{\mathcal{L}}_{J}^{v}(|\vec{q}|)|| J_{i}\right\rangle\right|^{2} \\
R_{v}^{\mathcal{M L}} & =\mathcal{R}\left[\left\langle J_{f}|| \widehat{\mathcal{L}}_{J}^{v}(|\vec{q}|)|| J_{i}\right\rangle\left\langle J_{f}|| \widehat{\mathcal{M}}_{J}^{v}(|\vec{q}|)|| J_{i}\right\rangle^{*}\right], \\
R_{v}^{T} & =\left[\left|\left\langle J_{f}|| \widehat{\mathcal{J}}_{J}^{\mathrm{mag}, v}(|\vec{q}|)|| J_{i}\right\rangle\right|^{2}+\left|\left\langle J_{f}|| \widehat{\mathcal{J}}_{J}^{\mathrm{el}, v}(|\vec{q}|)|| J_{i}\right\rangle\right|^{2}\right],
\end{aligned}
$$

$$
R_{v}^{T T}=\mathcal{R}\left[\left\langle J_{f}|| \widehat{\mathcal{J}}_{J}^{\mathrm{mag}, v}(|\vec{q}|)|| J_{i}\right\rangle\left\langle J_{f}|| \widehat{\mathcal{J}}_{J}^{\mathrm{el}, v}(|\vec{q}|)|| J_{i}\right\rangle^{*}\right] .
$$

Here $\widehat{\mathcal{M}}_{J}^{v}, \widehat{\mathcal{L}}_{J}^{v}, \widehat{\mathcal{J}}_{J}^{\mathrm{mag}, v}$ and $\widehat{\mathcal{J}}_{J}^{\mathrm{el}, v}$ are the Coulomb, longitudinal, transverse magnetic, and transverse electric operators, respectively $[46,47]$.

To calculate the nuclear response functions, we use the CRPA approach which is described in detail in Refs. [36-39]. Here we will briefly present the essence of our model. We start by describing the nucleus within a mean-field (MF) approximation. The MF potential is obtained by solving the Hartree-Fock (HF) equations with a Skyrme (SkE2) two-body interaction [36,37]. The sequential filling of the single-nucleon orbits automatically introduces Pauli blocking. The continuum wave functions are obtained by integrating the positive-energy Schrödinger equation with appropriate boundary conditions. In this manner, we account for the final-state interactions of the outgoing nucleon. Once we have bound and continuum single-nucleon wave functions, we introduce the long-range correlations through a CRPA approach. We solve the CRPA equations with a Green's function formalism. The RPA describes a nuclear excited state as the linear combination

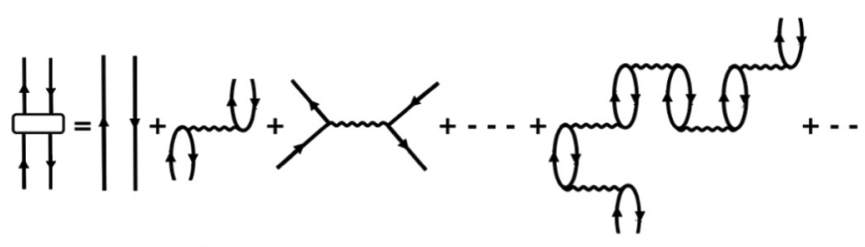

(a) (b)

(c)

(d)

FIG. 3. Diagrammatic representation of the polarization propagator $\Pi^{(\mathrm{RPA})}$ for particle-hole states. Panel (a) corresponds to the unperturbed polarization propagator $\Pi^{(0)}$, (b) and (c) are the first-order direct and exchange RPA diagrams, and (d) represents a typical higher-order RPA diagram.

of particle-hole $\left(p h^{-1}\right)$ and hole-particle $\left(h p^{-1}\right)$ excitations out of a correlated ground state

$$
\left|\Psi_{\mathrm{RPA}}^{C}\right\rangle=\sum_{C^{\prime}}\left[X_{C, C^{\prime}}\left|p^{\prime} h^{\prime-1}\right\rangle-Y_{C, C^{\prime}}\left|h^{\prime} p^{\prime-1}\right\rangle\right]
$$

where $C$ denotes the full set of quantum numbers representing an accessible channel. The Green's function approach allows one to treat the single-particle energy continuum exactly by treating the RPA equations in coordinate space. The RPA polarization propagator, obtained by the iteration of the firstorder contributions to the particle-hole Green's function, is written as

$$
\begin{aligned}
& \Pi^{(\mathrm{RPA})}\left(x_{1}, x_{2} ; E_{x}\right) \\
& =\Pi^{(0)}\left(x_{1}, x_{2} ; E_{x}\right)+\frac{1}{\hbar} \int d x d x^{\prime} \Pi^{0}\left(x_{1}, x ; E_{x}\right) \\
& \quad \times \tilde{V}\left(x, x^{\prime}\right) \Pi^{(\mathrm{RPA})}\left(x^{\prime}, x_{2} ; E_{x}\right),
\end{aligned}
$$

where $E_{x}$ is the excitation energy of the target nucleus and $x$ is a shorthand notation for the combination of the spatial, spin, and isospin coordinates. The $\Pi^{(0)}$ in Eq. (22) corresponds to the HF contribution to the polarization propagator and $\tilde{V}$ denotes the antisymmetrized nucleon-nucleon interaction. The $\mathrm{HF}$ responses can be retrieved by switching off the second term in the above equation. Figure 3 shows different components contributing to the polarization propagator.

A limitation of the RPA formalism is that the configuration space is restricted to $1 \mathrm{p}-1 \mathrm{~h}$ excitations. As a result only the escape-width contribution to the final-state interaction is accounted for and the spreading width of the particle states is neglected. This affects the description of giant resonances in the CRPA formalism. The energy location of the giant resonance is generally well predicted but the width is underestimated and the height of the response in the peak is overestimated. To remedy this, several methods have been proposed such as the folding procedure of Refs. [12,28,48,49]. Here, we use a simplified phenomenological approach where the modified response functions $R^{\prime}\left(q, \omega^{\prime}\right)$ are obtained after folding the HF and CRPA response functions $R(q, \omega)$ :

$$
R^{\prime}\left(q, \omega^{\prime}\right)=\int_{-\infty}^{\infty} d \omega R(q, \omega) L\left(\omega, \omega^{\prime}\right)
$$

with $L$ a Lorentzian

$$
L\left(\omega, \omega^{\prime}\right)=\frac{1}{2 \pi}\left[\frac{\Gamma}{\left(\omega-\omega^{\prime}\right)^{2}+(\Gamma / 2)^{2}}\right] .
$$



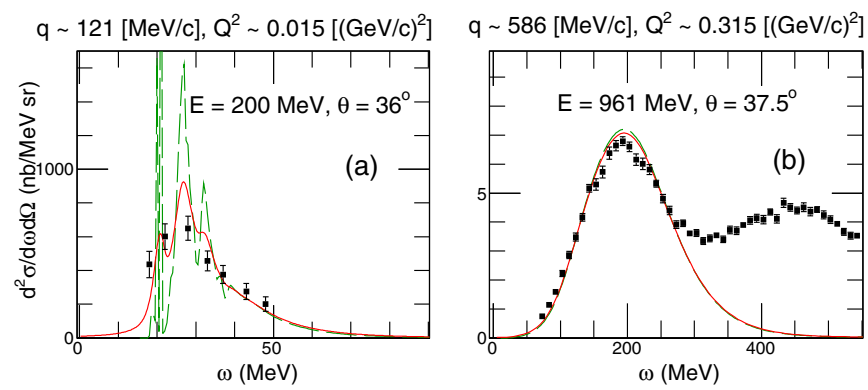

FIG. 4. (Color online) Comparison of ${ }^{12} \mathrm{C}\left(e, e^{\prime}\right)$ cross sections obtained with (full line) and without (dashed lines) the folding method. The experimental data are from (a) [51] and (b) [53].

We use an effective value of $\Gamma=3 \mathrm{MeV}$ which complies well with the predicted energy width in the giant-resonance region [48], where one expects the effect of the folding to be most important. The overall effect of folding is a redistribution of strength from peak to the tails. In line with the conclusions drawn in Refs. [12,20], the energy integrated response functions are not much affected by the folding procedure of Eq. (23). In Fig. 4, we compare the $\left(e, e^{\prime}\right)$ cross sections obtained with and without folding. Figure 4(a) clearly shows that in the giant-resonance region, the adopted folding procedure spreads the strength over a wider $\omega$ range, thereby considerably improving the quality of agreement with the data. At higher $\omega$ [Fig. 4(b)] the effect of the folding is marginal. All computed cross-section results shown in the paper adopt the folding procedure of Eq. (23).

Our approach is self-consistent because we use the same SkE2 interaction in both the HF and CRPA equations. The parameters of the momentum-dependent SkE2 force are optimized against ground-state and low-excitation energy properties [50]. Under those conditions the virtuality $Q^{2}$ of the nucleon-nucleon vertices is small. At high virtualities $Q^{2}$, the SkE2 force tends to be unrealistically strong. We remedy this by introducing a dipole hadronic form factor at the nucleon-nucleon interaction vertices

$$
V\left(Q^{2}\right) \rightarrow V\left(Q^{2}=0\right) \frac{1}{\left(1+\frac{Q^{2}}{\Lambda^{2}}\right)^{2}},
$$

where we introduced the free cutoff parameter $\Lambda$. We adopt $\Lambda=455 \mathrm{MeV}$, a value which is optimized in a $\chi^{2}$ test of the comparison of $A\left(e, e^{\prime}\right)$ CRPA cross sections with the experimental data of Refs. [51-58]. In the $\chi^{2}$ test, we consider the theory-experiment comparison from low values of $\omega$ up to the maximum of the quasielastic peak. We have restricted our fit to the low- $\omega$ side of the quasielastic peak, because the high- $\omega$ side is subject to corrections stemming from intermediate $\Delta$ excitation, which is not included in our model.

The influence of the nuclear Coulomb field on the lepton is taken into account by means of an effective momentum approximation (EMA) [45]. In order to take into account the reduced lepton wavelength, the three-momentum transfer is enhanced in an effective way

$$
q_{\mathrm{eff}}=q+1.5\left(\frac{Z^{\prime} \alpha \hbar c}{R}\right)
$$

where $R=1.24 A^{1 / 3} \mathrm{fm}$. The lepton wave functions are modified accordingly

$$
\Psi_{l}^{\mathrm{eff}}=\zeta\left(Z^{\prime}, E, q\right) \Psi_{l}
$$

with

$$
\zeta\left(Z^{\prime}, E, q\right)=\sqrt{\frac{q_{\mathrm{eff}} E_{\mathrm{eff}}}{q E},}
$$

where $E$ ( $\left.E_{\text {eff }}\right)$ is the energy (effective energy) of the outgoing lepton.

Our description of the nuclear dynamics is based on a nonrelativistic framework. For $q>500 \mathrm{MeV} / c$, the momentum of the emitted nucleon is comparable with its rest mass, and relativistic effects become important. We have implemented relativistic corrections in an effective fashion, as suggested in Refs. $[27,28,35]$. Those references show that a satisfactory description of relativistic effects can be achieved by following kinematic substitution in the nuclear response

$$
\lambda \rightarrow \lambda(1+\lambda),
$$

where $\lambda=\omega / 2 M_{N}$ and $M_{N}$ is the nucleon mass. The above substitution produces a reduction of the width of the one-body responses and a shift in the peak toward smaller values of $\omega$. The correction becomes sizable for $q \gtrsim 500 \mathrm{MeV} / c$.

\section{RESULTS}

To test our model, we start with the calculation of $\left(e, e^{\prime}\right)$ cross sections on different nuclei and the response functions for electron scattering off ${ }^{12} \mathrm{C}$, in Sec. III A. We confront our numerical results with the data of Refs. [51-59]. We discuss the neutrino-scattering results in Sec. III B.

\section{A. Electron scattering}

In this section, we present our results for the $\mathrm{QE} A\left(e, e^{\prime}\right)$ cross sections. For any given $E_{i}$, the nuclear response depends on $q^{\mu}$. Energy transfers below the particle knockout threshold result in nuclear excitations in discrete states. At slightly higher energies, the giant dipole resonance (GDR) shows up. Only at substantially higher energy can one distinguish the peak corresponding to QE one-nucleon knockout. In an ideal case, if an electron scatters from a free nucleon, one would expect a narrow peak at $\omega=Q^{2} / 2 M_{N}$. Deviations from that peak are due to the nuclear dynamics. The heavier the target nucleus, the wider the peak. The shift of the peak is due to nuclear binding and correlations.

For the two vector form factors entering in the responses, we use the standard dipole parametrization of Ref. [60]. In Fig. 5 we present results of our numerical calculations for ${ }^{12} \mathrm{C}\left(e, e^{\prime}\right)$. We compare CRPA and HF predictions with the measurements performed at the Saclay Linear Accelerator [51], Bates Linear Accelerator Center [52], Stanford Linear Accelerator Center [53,55], Yerevan electron synchrotron [54], and DESY [56]. The comparison is performed over a broad range of threeand four-momentum transfers: $95 \lesssim q \lesssim 1050 \mathrm{MeV} / c$, and $0.009 \lesssim Q^{2} \lesssim 0.900(\mathrm{GeV} / c)^{2}$. Our predictions are reasonably successful in describing the data over the broad kinematical range considered here. Moreover, they compare favorably 

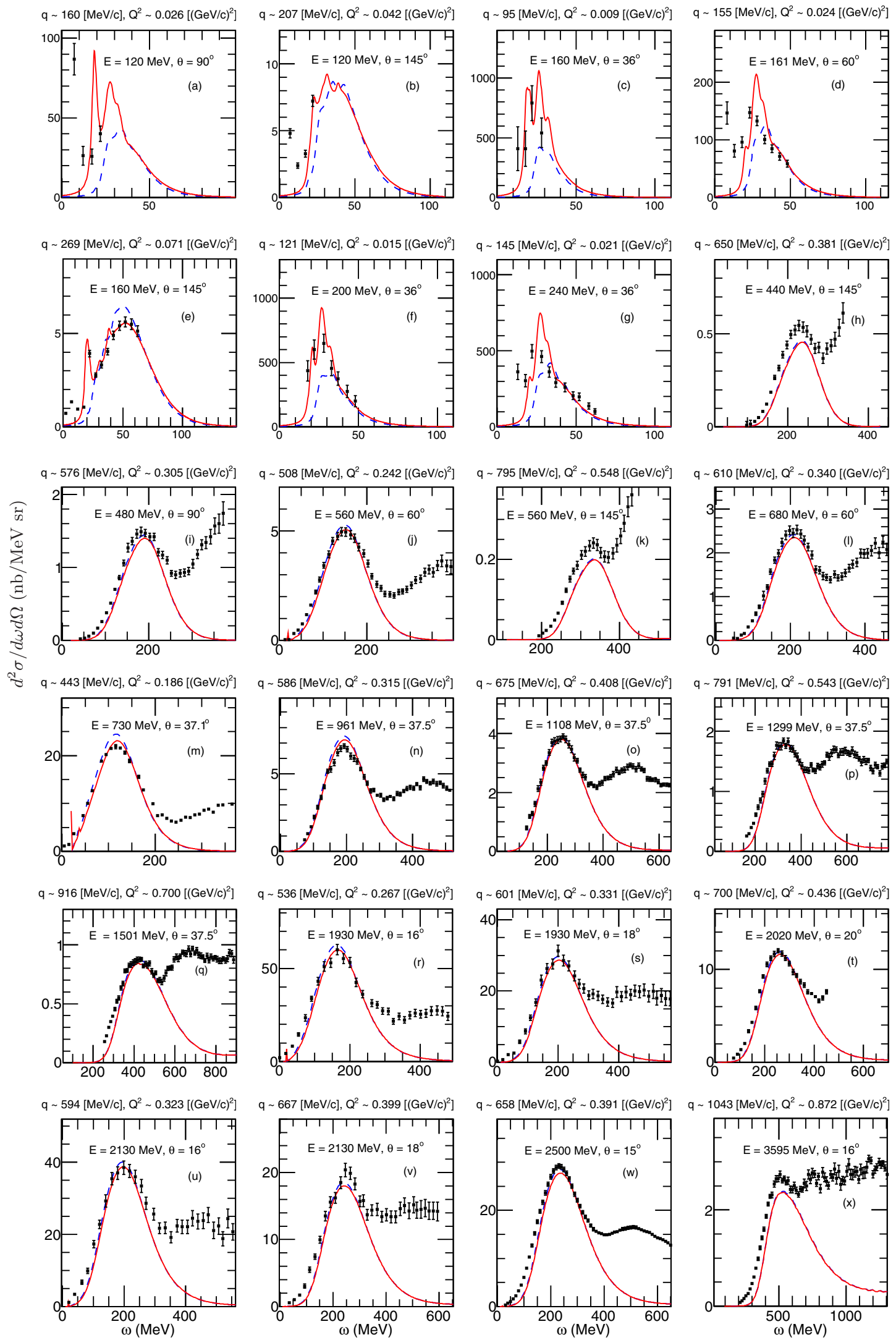

FIG. 5. (Color online) Double differential cross section for ${ }^{12} \mathrm{C}\left(e, e^{\prime}\right)$. CRPA (solid lines) and HF (dashed lines) cross sections are compared with the data of Refs. [51-56]. The $q$ and $Q^{2}$ values, at the top of each panel, are calculated at quasielastic conditions $Q^{2} /\left(2 M_{N} \omega\right)=1$, with $M_{N}$ the nucleon mass. 


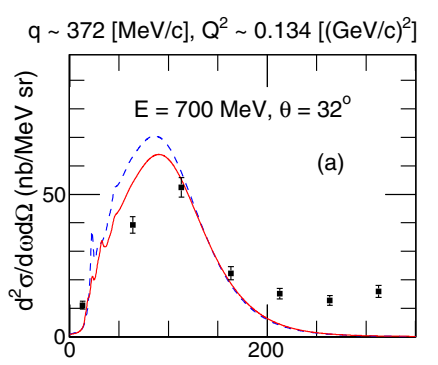

$\mathrm{q} \sim 447[\mathrm{MeV} / \mathrm{c}], \mathrm{Q}^{2} \sim 0.190\left[(\mathrm{GeV} / \mathrm{c})^{2}\right]$

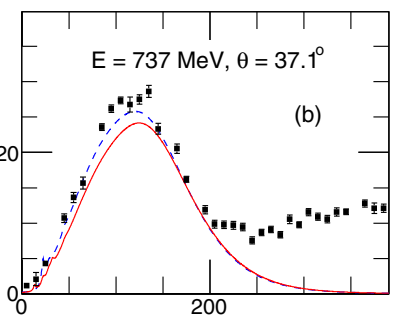

$\mathrm{q} \sim 466[\mathrm{MeV} / \mathrm{c}], \mathrm{Q}^{2} \sim 0.206\left[(\mathrm{GeV} / \mathrm{c})^{2}\right]$

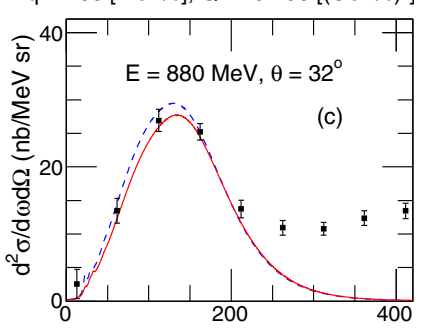

$\mathrm{q} \sim 572[\mathrm{MeV} / \mathrm{c}], \mathrm{Q}^{2} \sim 0.302\left[(\mathrm{GeV} / \mathrm{c})^{2}\right]$

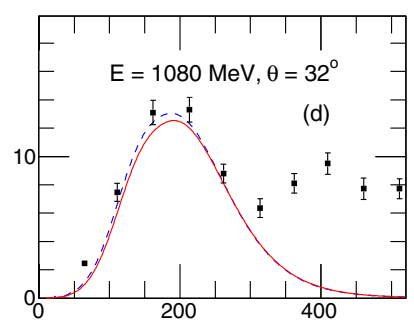

$\mathrm{q} \sim 636[\mathrm{MeV} / \mathrm{c}], \mathrm{Q}^{2} \sim 0.367\left[(\mathrm{GeV} / \mathrm{c})^{2}\right]$
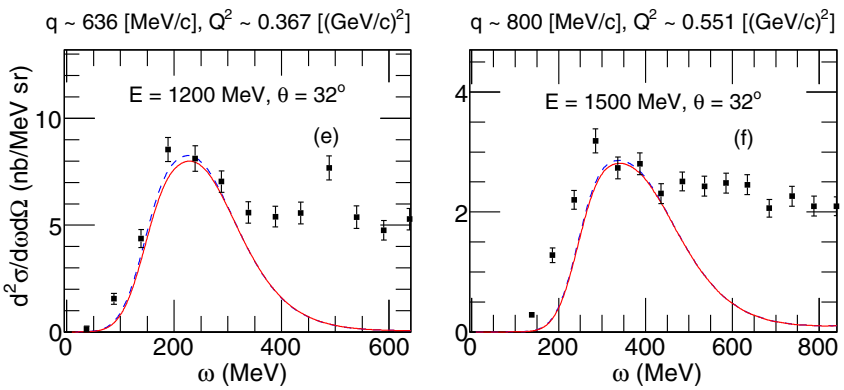

FIG. 6. (Color online) As in Fig. 5 but for ${ }^{16} \mathrm{O}\left(e, e^{\prime}\right)$. The data are from Refs. [52,57].

with the cross-section results of Refs. [26,30]. The interesting feature of our results is the prediction of the nuclear excitations at small energy $(\omega<50 \mathrm{MeV})$ and momentum transfers $(q<300 \mathrm{MeV} / c)$, well below the QE peak. This feature can be appreciated in Figs. 5(a) $-5(\mathrm{~g})$. The HF and CRPA $\mathrm{A}\left(e, e^{\prime}\right)$ cross sections are identical for $Q^{2} \gtrsim 0.25(\mathrm{GeV} / c)^{2}$. The cross section drops by two orders of magnitude with the shift in scattering angle from $36^{\circ}$ to $145^{\circ}$, for a fixed energy, as evident from Figs. 5(c)-5(e) for an incoming energy of $160 \mathrm{MeV}$. Even for higher incoming electron energies the cross-section measurements at smaller scattering angles are still dominated by QE processes. Obviously, the measured cross sections include contributions from channels beyond $\mathrm{QE}$, like $\Delta$ excitations, evident as the second peak in the data, and $2 \mathrm{p}-2 \mathrm{~h}$ contributions. Our description is restricted to $\mathrm{QE}$ processes and further work is in progress on the role of processes beyond $\mathrm{QE}$ ones [61].

The double differential ${ }^{16} \mathrm{O}\left(e, e^{\prime}\right)$ cross sections are shown in Fig. 6. Our numerical calculations reasonably describe the QE parts of the measurements performed at ADONE [57] and at the Bates Linear Accelerator Center [52]. Further, the calculations for the heavier target ${ }^{40} \mathrm{Ca}$ are presented in Fig. 7 . Again, the comparison with the experimental data taken at Bates Linear Accelerator Center [58] is fair.
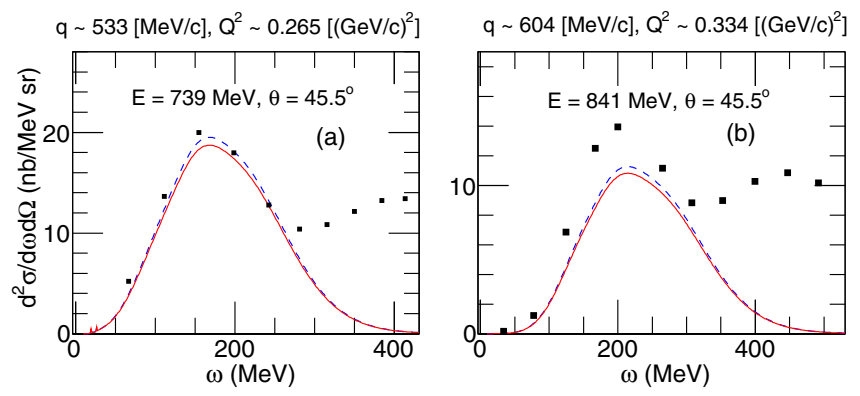

FIG. 7. (Color online) Same as Figs. 5 and 6 but on a ${ }^{40} \mathrm{Ca}$ target, the measurements are from Ref. [58].

In Fig. 8 we compare cross sections obtained with two different parametrizations of the single-nucleon wave functions and nucleon-nucleon residual interactions. The Landau Migdal (LM) [62] and SKE2 [37,50] yield similar cross sections while the use of the Woods-Saxon (WS) [34] wave function slightly shifts and reduces the strength of the cross section. This can be attributed to the fact that the HF wave functions have larger high-momentum components than the WS ones.

The $\left(e, e^{\prime}\right)$ cross section receives contributions from the longitudinal and transverse components, as can be seen in Eq. (3). A separation of these two response functions provides further detail about the target dynamics. It is worth mentioning that the experimental values of responses are extracted from a set of cross-section measurements using a Rosenbluth separation [63]. The data of Ref. [59] is determined by a reanalysis of the world data on $\left(e, e^{\prime}\right)$ cross sections. Interestingly, that resulted in a significant difference from the measurements of Ref. [51], as can be seen in Fig. 9(b). The comparison between our predictions on ${ }^{12} \mathrm{C}$ with the experimental data of Refs. [51,59] is quite satisfactory. The longitudinal responses are overestimated and the transverse responses are usually underestimated. Our predictions are in line with those predicted in Ref. [59] and with the continuum shell model predictions of Ref. [64]. It is long known that the inclusion of processes involving meson exchange current are needed to account for the transverse strength of the electromagnetic response $[65,66]$. The calculations carried out on light nuclei overwhelmingly suggest that single-nucleon knockout processes, such as in this work, are dominant
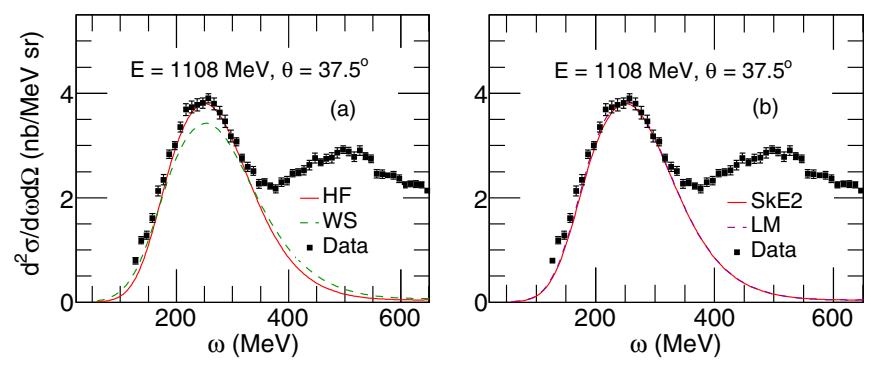

FIG. 8. (Color online) Comparison of cross sections obtained (on ${ }^{12} \mathrm{C}$ ) (a) with the HF and WS single-particle wave functions with SKE2 as residual interaction and (b) with the SKE2 and LM residual interaction with $\mathrm{HF}$ as single-particle wave functions. 

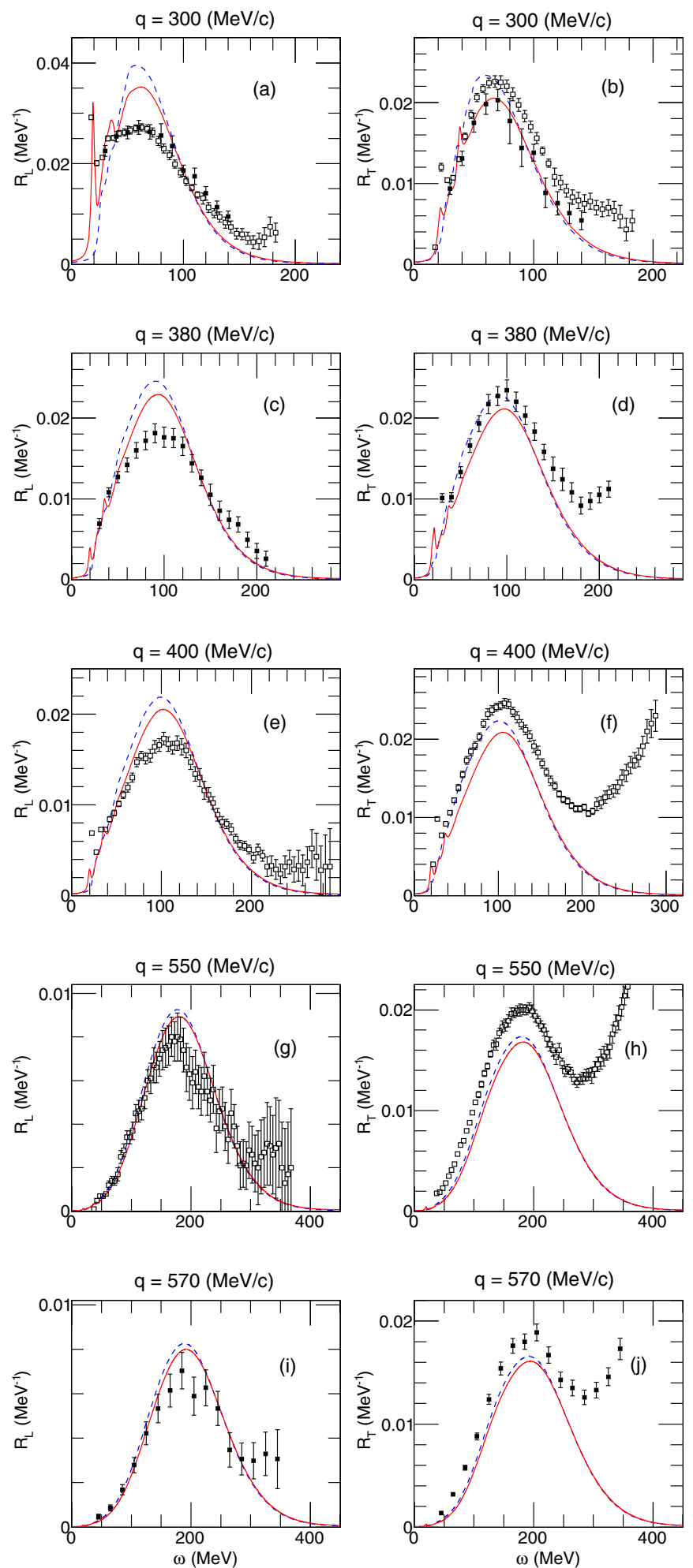

FIG. 9. (Color online) Longitudinal and transverse responses for ${ }^{12} \mathrm{C}\left(e, e^{\prime}\right)$ for different values of $q$. Solid lines are CRPA predictions and dashed lines are HF predictions. Experimental data are from Ref. [59] (filled squares) and Ref. [51] (open squares).

in the longitudinal channel while in the transverse channel two-nucleon processes provide substantial contributions.

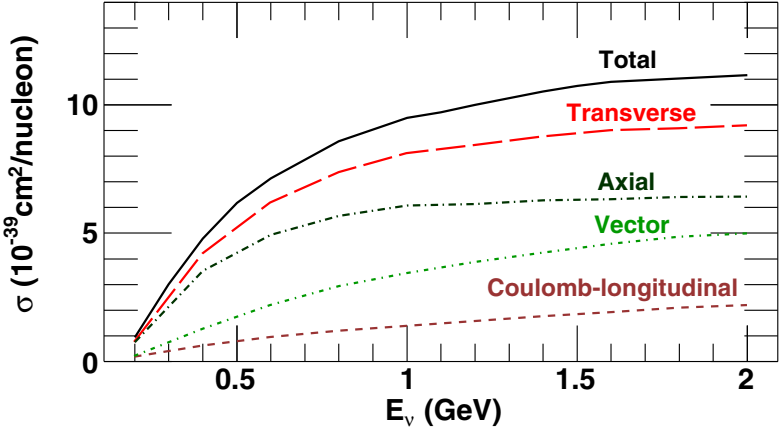

FIG. 10. (Color online) Different contributions to the total ${ }^{12} \mathrm{C}\left(v_{\mu}, \mu^{-}\right)$cross section (per neutron) as a function of incoming neutrino energy. The sum of transverse and Coulomb-longitudinal (axial and vector) is the total cross section.

\section{B. Neutrino scattering}

The calculation of ${ }^{12} \mathrm{C}\left(v_{l}, l^{-}\right)$response functions involve two vector form factors and one axial form factor. We use the BBBA05 parametrization of Ref. [67] for the two vector form factors, and the standard dipole parametrization of the axial form factor with $M_{A}=1.03 \pm 0.02 \mathrm{GeV}$ [68-70].

In Fig. 10 we display different contributions to the total ${ }^{12} \mathrm{C}\left(v_{\mu}, \mu^{-}\right)$cross section, as a function of the incoming neutrino energy. The axial contribution is larger than the vector one. Related to this, neutrino cross sections are dominated by the transverse current.

Electron-scattering cross-section measurements are typically performed for a fixed incoming electron energy and scattering angle. As neutrinos are produced as the secondary products of a decaying primary beam, the interacting neutrino's energy is not sharply defined. The initial neutrino energy is reconstructed using the kinematics of the final outgoing lepton. This is a major source of uncertainty whereby nuclear structure can have an important influence.

The neutrino flux in oscillation experiments typically covers a wide energy range from about $100 \mathrm{MeV}$ to a few $\mathrm{GeV}$. The cross section measured at a single energy and scattering angle of the outgoing lepton picks up contributions from scattering processes at different energies, with varying weights. In Fig. 11, we show the differential cross section (in outgoing

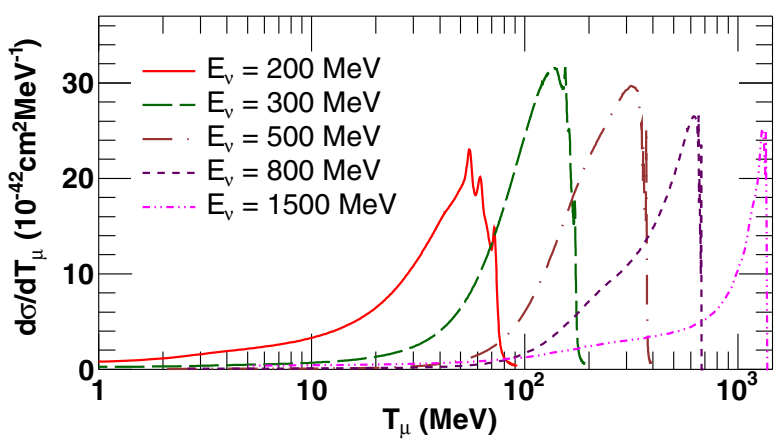

FIG. 11. (Color online) Cross section for ${ }^{12} \mathrm{C}\left(v_{\mu}, \mu^{-}\right)$as a function of outgoing muon kinetic energy $T_{\mu}$, for different incoming neutrino energies. Note the log scale on the horizontal axis. 

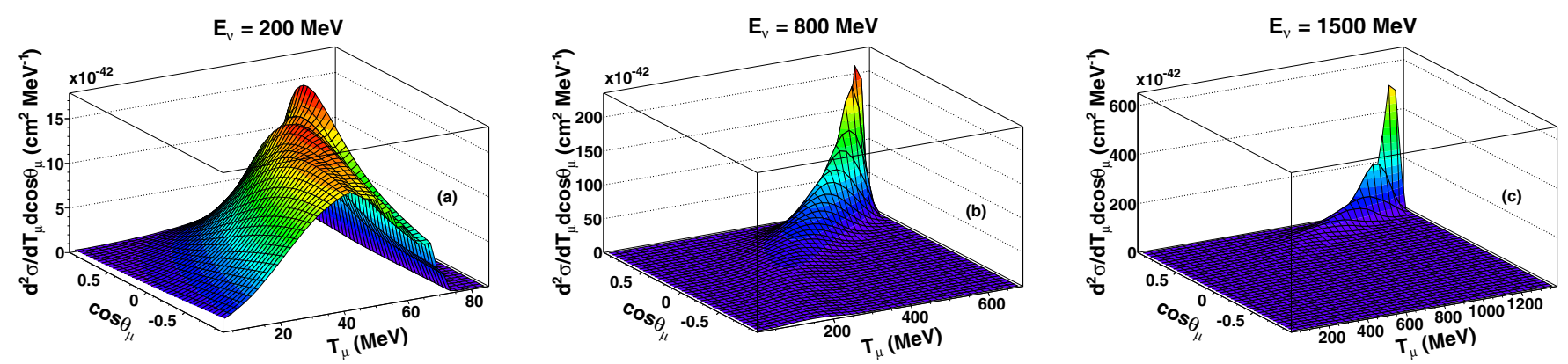

FIG. 12. (Color online) Double differential ${ }^{12} \mathrm{C}\left(v_{\mu}, \mu^{-}\right)$cross sections plotted as a function of $T_{\mu}$ and $\cos \theta_{\mu}$, for three neutrino energies.

muon energy) for $200 \lesssim E_{v} \lesssim 1500 \mathrm{MeV}$. It is evident from the figure that with increasing $E_{v}$ the strengths of the cross sections shift in muon energy. Also, there is a clear signature of the low- $\omega$ excitations even at neutrino energies around the peak of the MiniBooNE and T2K $v_{\mu}$ spectra.

The measured cross sections are flux-folded double differential in outgoing muon kinetic energy $T_{\mu}$ and scattering angle $\cos \theta_{\mu}$. To illustrate the low-energy excitations and general behavior of double differential ${ }^{12} \mathrm{C}\left(v_{\mu}, \mu^{-}\right)$cross sections at fixed energies, we display in Fig. 12 the double differential cross sections for $E_{v}=200,800$, and $1500 \mathrm{MeV}$. With the increase in incoming neutrino energy, the strength shifts in the forward direction and the width of giant resonances reduces. In Fig. 13, we plot the double differential cross section at different fixed values of $\cos \theta_{\mu}$. For $E_{v}=150 \mathrm{MeV}$, the double differential cross section is dominated by low-lying nuclear excitations, as evident from Fig. 13(a). For neutrino energies around the mean energy of the MiniBooNE [1] and T2K [6] fluxes, $E_{v}=800 \mathrm{MeV}$ [Fig. 13(c)], the nuclear collective excitations are still sizable at forward muon scattering angles. The same feature is still visible for very forward scattering
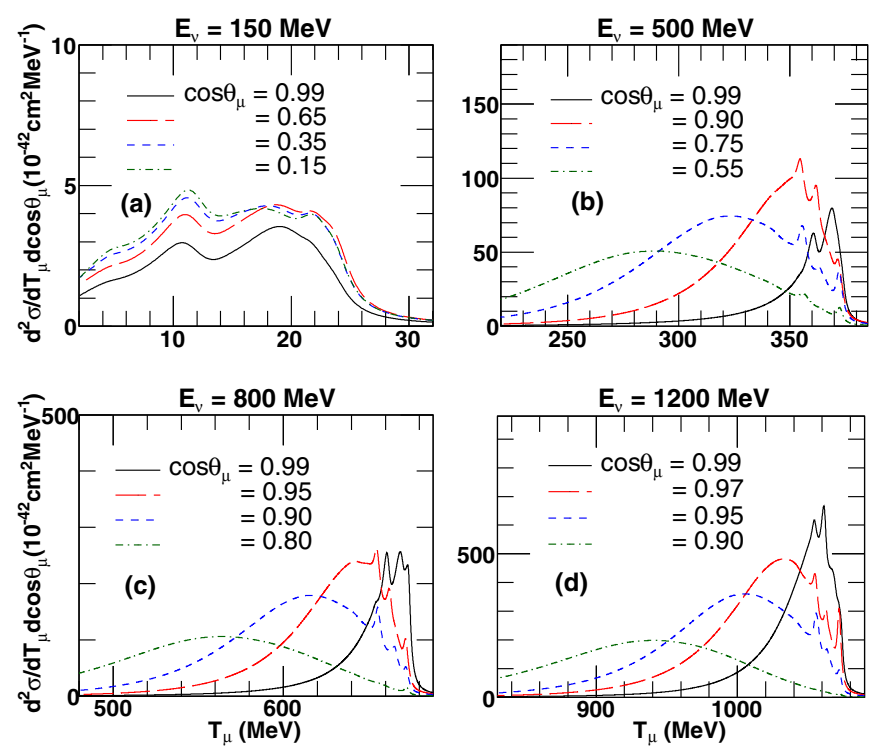

FIG. 13. (Color online) Low-energy excitations in double differential cross sections for ${ }^{12} \mathrm{C}\left(v_{\mu}, \mu^{-}\right)$plotted as a function of $T_{\mu}$, for different $\cos \theta_{\mu}$ values. off neutrinos with an energy of $1200 \mathrm{MeV}$ [Fig. 13(d)]. The contribution of collective excitations to neutrino-nucleus responses cannot be accounted for within the RFG-based simulation codes. As evident from the results presented here, they can account for non-negligible contributions to the signal even at higher neutrino energies.

In Fig. 14, we show the transverse and Coulomblongitudinal contribution to the double differential cross sections. For $\cos \theta_{\mu}=0.99$, the Coulomb-longitudinal contribution of the quasielastic cross section is comparable to the transverse one. The transverse contribution dominates the cross section as soon as one moves away from the very forward direction. This feature along with the giant-resonance contribution to forward-scattering cross sections accounts for most of the strength at very small momentum transfers. Theoretical models, which do not predict this behavior, tend to underestimate the cross section for forward-scattering angles, as discussed in Ref. [71].

\section{CONCLUSIONS}

We presented a detailed discussion of CRPA predictions for quasielastic electron-nucleus and neutrino-nucleus responses. We assessed inclusive quasielastic electron-nucleus cross sections on ${ }^{12} \mathrm{C}$, ${ }^{16} \mathrm{O}$, and ${ }^{40} \mathrm{Ca}$. We consider momentum transfers over the broad range $95 \lesssim q \lesssim 1050 \mathrm{MeV} / c$ in combination with energy transfers which favor the quasielastic nucleon-knockout reaction process. We confronted our predictions with high-precision electron-scattering data. We separated the longitudinal and transverse responses on ${ }^{12} \mathrm{C}$, for $300 \lesssim q \lesssim 570 \mathrm{MeV} / c$, and compared them with the
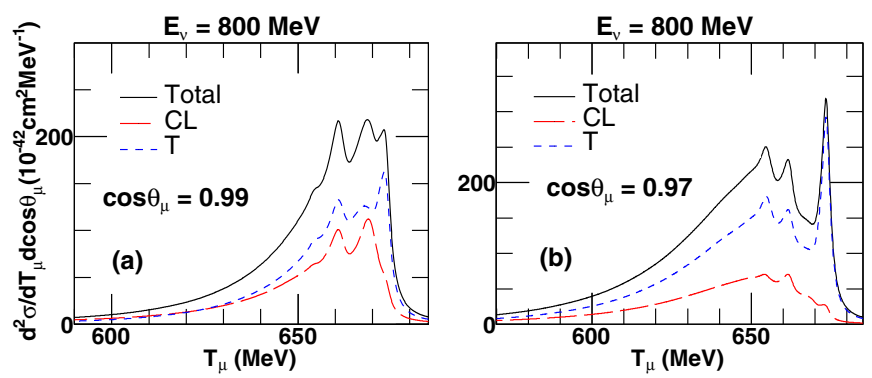

FIG. 14. (Color online) Coulomb-longitudinal (CL) and transverse $(\mathrm{T})$ contributions to the double differential cross sections, at $E_{v}=800 \mathrm{MeV}$ and two values of $\cos \theta_{\mu}$. 
data. A reasonable overall description of the data, especially those corresponding with low-energy nuclear excitations, is reached.

We calculated ${ }^{12} \mathrm{C}\left(v_{\mu}, \mu^{-}\right)$cross sections, relevant for accelerator-based neutrino-oscillation experiments. We illustrated how low-energy nuclear excitations are induced by neutrinos. We paid special attention to contributions where nuclear-structure details become important but remain unobserved in RFG-based models. We show that low-energy excitations can account for non-negligible contributions to the signal of accelerator-based neutrino-oscillation experiments, especially at forward neutrino-nucleus Scattering.

\section{ACKNOWLEDGMENTS}

We thank Luis Alvarez-Ruso and Teppei Katori for useful discussions. This research was funded by the Interuniversity Attraction Poles Programme initiated by the Belgian Science Policy Office, the Research Foundation Flanders (FWOFlanders) and by the Erasmus Mundus External Cooperations Window's Eurindia Project.
[1] A. A. Aguilar-Arevalo et al. (MiniBooNE Collaboration), Phys. Rev. D 81, 092005 (2010).

[2] A. A. Aguilar-Arevalo et al. (MiniBooNE Collaboration), Phys. Rev. D 88, 032001 (2013).

[3] A. A. Aguilar-Arevalo et al. (MiniBooNE Collaboration), Phys. Rev. D 82, 092005 (2010).

[4] G. A. Fiorentini et al. (MINERvA Collaboration), Phys. Rev. Lett. 111, 022502 (2013).

[5] L. Fields et al. (MINERvA Collaboration), Phys. Rev. Lett. 111, 022501 (2013).

[6] K. Abe et al. (T2K Collaboration), Phys. Rev. D 87, 092003 (2013).

[7] K. Abe et al. (T2K Collaboration), Phys. Rev. D 90, 052010 (2014).

[8] J. G. Morfin, J. Nieves, and J. T. Sobczyk, Adv. High Energy Phys. 2012, 934597 (2012).

[9] L. Alvarez-Ruso, Y. Hayato, and J. Nieves, New J. Phys. 16, 075015 (2014).

[10] E. Kolbe, K. Langanke, F. K. Thielemann, and P. Vogel, Phys. Rev. C 52, 3437 (1995).

[11] C. Volpe, N. Auerbach, G. Colo, T. Suzuki, and N. Van Giai, Phys. Rev. C 62, 015501 (2000).

[12] A. Botrugno and G. Co', Nucl. Phys. A 761, 200 (2005).

[13] M. Martini, G. Co', M. Anguiano, and A. M. Lallena, Phys. Rev. C 75, 034604 (2007).

[14] A. R. Samana, F. Krmpotic, N. Paar, and C. A. Bertulani, Phys. Rev. C 83, 024303 (2011).

[15] http://www-boone.fnal.gov/

[16] http://t2k-experiment.org/

[17] W. M. Alberico, M. Ericson, and A. Molinari, Nucl. Phys. A 379, 429 (1982).

[18] M. Martini, M. Ericson, G. Chanfray, and J. Marteau, Phys. Rev. C 80, 065501 (2009).

[19] M. Martini, J. Phys. Conf. Ser. 408, 012041 (2013).

[20] A. Gil, J. Nieves, and E. Oset, Nucl. Phys. A 627, 543 (1997).

[21] J. Nieves, J. E. Amaro, and M. Valverde, Phys. Rev. C 70, 055503 (2004).

[22] J. Nieves, M. Valverde, and M. J. Vicente Vacas, Phys. Rev. C 73, 025504 (2006).

[23] O. Benhar, A. Fabrocini, S. Fantoni, and I. Sick, Nucl. Phys. A 579, 493 (1994).

[24] O. Benhar, N. Farina, H. Nakamura, M. Sakuda, and R. Seki, Phys. Rev. D 72, 053005 (2005).

[25] H. Nakamura, T. Nasu, M. Sakuda, and O. Benhar, Phys. Rev. C 76, 065208 (2007).
[26] A. M. Ankowski, O. Benhar, and M. Sakuda, Phys. Rev. D 91, 033005 (2015).

[27] J. E. Amaro, M. B. Barbaro, J. A. Caballero, T. W. Donnelly, and C. Maieron, Phys. Rev. C 71, 065501 (2005).

[28] J. E. Amaro, M. B. Barbaro, J. A. Caballero, T. W. Donnelly, and J. M. Udias, Phys. Rev. C 75, 034613 (2007).

[29] T. Leitner, O. Buss, L. Alvarez-Ruso, and U. Mosel, Phys. Rev. C 79, 034601 (2009).

[30] A. V. Butkevich and S. P. Mikheyev, Phys. Rev. C 72, 025501 (2005).

[31] A. V. Butkevich and S. A. Kulagin, Phys. Rev. C 76, 045502 (2007).

[32] A. Meucci, F. Capuzzi, C. Giusti, and F. D. Pacati, Phys. Rev. C 67, 054601 (2003).

[33] A. Meucci, C. Giusti, and F. D. Pacati, Nucl. Phys. A 739, 277 (2004).

[34] G. Co' and S. Krewald, Phys. Lett. B 137, 145 (1984).

[35] S. Jeschonnek and T. W. Donnelly, Phys. Rev. C 57, 2438 (1998).

[36] J. Ryckebusch, M. Waroquier, K. Heyde, J. Moreau, and D. Ryckbosch, Nucl. Phys. A 476, 237 (1988).

[37] J. Ryckebusch, K. Heyde, D. Van Neck, and M. Waroquier, Nucl. Phys. A 503, 694 (1989).

[38] N. Jachowicz, S. Rombouts, K. Heyde, and J. Ryckebusch, Phys. Rev. C 59, 3246 (1999).

[39] N. Jachowicz, K. Heyde, J. Ryckebusch, and S. Rombouts, Phys. Rev. C 65, 025501 (2002).

[40] N. Jachowicz, K. Vantournhout, J. Ryckebusch, and K. Heyde, Phys. Rev. Lett. 93, 082501 (2004).

[41] N. Jachowicz and G. C. McLaughlin, Phys. Rev. Lett. 96, 172301 (2006).

[42] N. Jachowicz, C. Praet, and J. Ryckebusch, Acta Physica Polonica B 40, 2559 (2009).

[43] N. Jachowicz and V. Pandey, AIP Conf. Proc. 1663, 050003 (2015).

[44] V. Pandey, N. Jachowicz, J. Ryckebusch, T. Van Cuyck, and W. Cosyn, Phys. Rev. C 89, 024601 (2014).

[45] J. Engel, Phys. Rev. C 57, 2004 (1998).

[46] J. S. O'Connell, T. W. Donnelly, and J. D. Walecka, Phys. Rev. C 6, 719 (1972).

[47] J. D. Walecka, Theoretical Nuclear and Subnuclear Physics (Oxford University, Oxford, 1995).

[48] R. D. Smith and J. Wambach, Phys. Rev. C 38, 100 (1988).

[49] A. DePace and M. Viviani, Phys. Rev. C 48, 2931 (1993).

[50] M. Waroquier, J. Ryckebusch, J. Moreau, K. Heyde, N. Blasi, S. Y. Van de Werf, and G. Wenes, Phys. Rep. 148, 249 (1987). 
[51] P. Barreau et al., Nucl. Phys. A 402, 515 (1983).

[52] J. S. O'Connell et al., Phys. Rev. C 35, 1063 (1987).

[53] R. M. Sealock et al., Phys. Rev. Lett. 62, 1350 (1989).

[54] D. S. Bagdasaryan et al., YERPHI-1077-40-88, 1988 (unpublished).

[55] D. B. Day et al., Phys. Rev. C 48, 1849 (1993).

[56] D. Zeller, DESY-F23-73-2, 1973 (unpublished).

[57] M. Anghinolfi et al., Nucl. Phys. A 602, 405 (1996).

[58] C. F. Williamson et al., Phys. Rev. C 56, 3152 (1997).

[59] J. Jourdan, Nucl. Phys. A 603, 117 (1996).

[60] E. J. Beise and R. D. McKeown, Comments Nucl. Part. Phys. 20, 105 (1991).

[61] T. Van Cuyck, N. Jachowicz, M. Martini, V. Pandey, and J. Ryckebusch (unpublished).

[62] G. Có and S. Krewald, Nucl. Phys. A 433, 392 (1985).
[63] M. N. Rosenbluth, Phys. Rev. 79, 615 (1950).

[64] J. E. Amaro, G. Co', and A. M. Lallena, arXiv:nucl-th/9902072.

[65] T. W. Donnelly, J. W. Van Orden, T. De Forest, Jr., and W. C. Hermans, Phys. Lett. B 76, 393 (1978).

[66] W. M. Alberico, M. Ericson, and A. Molinari, Ann. Phys. (NY) 154, 356 (1984).

[67] R. Bradford, A. Bodek, H. Budd, and J. Arrington, Nucl. Phys. Proc. Suppl. 159, 127 (2006).

[68] J. Beringer et al. (Particle Data Group), Phys. Rev. D 86, 010001 (2012).

[69] C. Amsler et al. (Particle Data Group), Phys. Lett. B 667, 1 (2008).

[70] V. Bernard, L. Elouadrhiri, and U. G. Meissner, J. Phys. G 28, R1 (2002).

[71] M. Martini and M. Ericson, Phys. Rev. C 90, 025501 (2014). 